\title{
Naive helper $T$ cells from BCG-vaccinated volunteers produce IFN- $\gamma$ and IL-5 to mycobacterial antigen-pulsed dendritic cells
}

\author{
Magdalena Kowalewicz-Kulbat ${ }^{1}$, Dominik Kaźmierczak ${ }^{1}$, Stefan Donevski ${ }^{1}$, \\ Franck Biet ${ }^{2}$, Joël Pestel ${ }^{3}$ and Wieslawa Rudnicka ${ }^{1}$ \\ ${ }^{1}$ Department of Immunology and Infectious Biology, University of Lodz, Poland \\ ${ }^{2}$ UR1282 Infectiologie Animale, Santé Publique (IASP-311), INRA Centre de Tours, Nouzilly, France \\ ${ }^{3}$ FRE2933 CNRS Université des Sciences et Technologies de Lille, France
}

\begin{abstract}
Mycobacterium bovis bacillus Calmette-Guérin (BCG) is a live vaccine that has been used in routine vaccination against tuberculosis for nearly 80 years. However, its efficacy is controversial. The failure of BCG vaccination may be at least partially explained by the induction of poor or inappropriate host responses. Dendritic cells (DCs) are likely to play a key role in the induction of immune response to mycobacteria by polarizing the reactivity of T lymphocytes toward a Th1 profile, contributing to the generation of protective cellular immunity against mycobacteria. In this study we aimed to investigate the production of Th1 and Th2 cytokines by naive $\mathrm{CD} 4^{+} \mathrm{T}$ cells to mycobacterial antigen-pulsed DCs in the group of young, healthy BCG vaccinated volunteers. The response of naive helper T cells was compared with the response of total blood lymphocytes. Our present results clearly showed that circulating naive CD45RA ${ }^{+} \mathrm{CD} 4^{+}$lymphocytes from BCG-vaccinated subjects can become effector helper cells producing IFN- $\gamma$ and IL-5 under the stimulation by autologous dendritic cells presenting mycobacterial protein antigen-PPD or infected with live $M$. bovis BCG bacilli.
\end{abstract}

Key words: Dendritic cells - T cells - IFN- $\gamma$ - IL-5 - BCG vaccine

\section{Introduction}

Dendritic cells (DCs) are professional antigen-presenting cells (APC) characterized by the expression of major histocompatibility complex (MHC) class I and II antigens, spontaneously clustering T cells and stimulating naive CD4 and CD8 T cells. These unique bone-marrow derived leucocytes are the most effective APC initiating a primary immune response to specific antigens or microbes invading the organism [1,2]. Dendritic cells differ from other APC such as B cells, monocytes and macrophages by their unique capacity to stimulate naive $\mathrm{T}$ cells in vivo and in vitro and to induce the polarization of immune response toward a Th1 or Th2 profile $[3,4]$. It is known that antigen-specific $\mathrm{CD}^{+}$and $\mathrm{CD} 8^{+} \mathrm{T}$ cells exist at very low fre-

Correspondence: W. Rudnicka, Dept. of Immunology and Infectious Biology; University of Łódż; Banacha 12/16, 90-237 Łódź, Poland; tel.: (+4842) 6354472,

fax.: (+4842) 6355818, e-mail: rudw@biol.uni.lodz.pl quencies in naive hosts. However, after being stimulated with antigen loaded dendritic cells, naive $\mathrm{CD} 4^{+} \mathrm{T}$ cells differentiate into functional helper T cell subsets called Th1, Th2 or Th17 cells depending largely on the surrounding cytokines $[5,6]$. The effector Th1 cells produce IFN- $\gamma$ and Th2 cells-IL-4 and IL-5. Th1 cells are essential for the protection against a variety of intracellular infections including mycobacteria.

A live attenuated strain of Mycobacterium bovis $\mathrm{BCG}$ is known as a vaccine against tuberculosis. Three billion people have been vaccinated with BCG worldwide. Mycobacterium bovis BCG induces a Th1-type immune response upon the infection of the host macrophages. Among the Th1 cytokines that are induced by BCG infection, IFN- $\gamma$ plays a major role in the activation of cell-mediated immunity $[7,8]$. The transfer of BCG-infected DCs into mice led to rapid IFN- $\gamma$ responses against mycobacterial antigens [9]. Various in vitro and in vivo studies demonstrated that the interactions of dendritic cells with mycobacteria play a key role in immunity to these bacilli [10]. It has 
been documented that priming and activation of mycobacterial antigen-specific $\mathrm{T}$ lymphocytes are essential for protection against tuberculosis. Moreover, in murine model of the infection with $M$. bovis BCG, DCs were the major leukocyte population involved in early activation of naive T lymphocytes [10]. There are some studies which indicate the effect of mycobacteria on DC phenotype, maturation and the profile of released cytokines. Viable BCG mycobacteria induce the maturation of DCs resulting in the up-regulation of $\mathrm{MHC}$ and costimulatory molecules on the surface of $\mathrm{DC}$ and in priming of naive T cells [10-13]. Although the maturation of DCs in response to mycobacteria, such as Mycobacterium bovis BCG is well described the subsequent response of autologous $\mathrm{T}$ cells to mycobacterium-infected DC still remains unclear. There is a general consensus that BCG vaccination is associated with lower incidence of tuberculosis in children and protection against severe forms of childhood meningeal and miliary tuberculosis $[14,15]$. On the other hand, numerous controlled clinical trials yielded diverse and contradictory results on protecting adults from tuberculosis by $B C G$ vaccination. They suggested that BCG driven protective immunity abates with the age and a booster tuberculosis BCG vaccine is needed to induce a greater protection against virulent mycobacteria in adults neonataly vaccinated with BCG. Thus in this study, we investigated the Th1 and $\mathrm{Th} 2$ cytokine responses of naive $\mathrm{T} \mathrm{CD} 4^{+}$lymphocytes to mycobacterial antigens presented by autologous DC in the group of young healthy volunteers who had been subjected to BCG vaccinations at birth and school age. The responses of peripheral blood naive $\mathrm{T}$ helper cells were compared with the responses of total lymphocyte population.

\section{Materials and methods}

Blood donors. Blood was collected from 38 young healthy volunteers aged 23-30. All participants had been vaccinated with BCG according to state policy. They were subjected to neonatal BCG vaccination. BCG revaccinations were given at school age to those who were tuberculin negative after Mantoux testing with PPD (purified protein derivative). The study was approved by the Human Ethics Committee.

Cell preparations. After depletion of platelet-rich plasma, peripheral blood was diluted in RPMI 1640 (1:1 Biomed, Lublin, Poland) and deposited over a Ficoll density gradient (Amersham Biosciences). After centrifugation of $400 \mathrm{~g}$ for 30 minutes, human PBMCs (peripheral blood mononuclear cells) were isolated, washed and resuspended in PBS, $\mathrm{pH}=7.2$, supplemented with $0.5 \%$ BSA (Sigma, Poland) and $2 \mathrm{mM}$ EDTA. PBMCs were incubated on ice for 30 minutes with anti-human CD14 antibodies coupled to magnetic micro-beads (Miltenyi Biotec). After elution, CD14 cells were resuspended in RPMI 1640 containing $10 \%$ heat-inactivated FCS (Cytogen, Poland), 2mM L-glutamine, and antibiotics (penicillin, streptomycin $1 \%$; Sigma) before plating $\left(3 \times 10^{6}\right.$ cells $/ 3$ $\mathrm{ml}$ per well) into 6-well flat bottomed culture plates. $\mathrm{CD} 14^{+}$cells were differentiated into monocyte-derived dendritic cells (MD-
DCs) for 6 days in the medium supplemented with GM-CSF (25 ng/ml; R\&D Systems, United Kingdom) and IL-4 (200 IU/ml; R\&D Systems, United Kingdom). MD-DCs were washed before being used for cocultures with $\mathrm{T}$ cells.

Naive $\mathrm{CD} 45 \mathrm{RA}^{+} \mathrm{CD}^{+}$were isolated from the eluted CD14cell fraction by using a naive $\mathrm{CD} 4^{+} \mathrm{T}$-cell isolation kit (containing human CD8, CD11b, CD16, CD19, CD36, CD56 and CD45RO antibodies, Miltenyi Biotec). Naive T cells were frozen in FCS containing 10\% DMSO until used.

Total lymphocyte population containing all types of $\mathrm{T}$ cells, B cells, NK (natural killer) cells was obtained as the eluted CD14cell fraction after magnetic separation and frozen in FCS containing $10 \%$ DMSO until used.

Preparation of bacteria. Mycobacterium bovis BCG bacilli (strain 1173P2; World Health Organisation) used for the stimulation of DC was from the cultures in liquid Middlebrook $7 \mathrm{H} 9$ grown at $37 \mathrm{C}$ under a humidified $5 \% \mathrm{CO}_{2}$ atmosphere for 2 weeks. Bacterial suspensions were prepared by dispersing BCG in PBS and vortexed. The density of bacterial suspension was estimated by counting colony-forming units by the standard viable count technique on Middlebrook 7H10 agar plates.

Activation of DCs. MD-DCs $\left(1 \times 10^{5}\right.$ cells $/ \mathrm{ml}$ per well $)$ were incubated for $24 \mathrm{~h}$ with PPD $(10 \mu \mathrm{g} / \mathrm{ml})$ (Purified Protein Derivative; Statens Serum Institut, Kopenhagen, Denmark) or with BCG at ratio 1 bacteria per DC). As a positive control, DCs were stimulated with LPS ( $1 \mu \mathrm{g} / \mathrm{ml}$; Sigma, Poland).

Cytokine assay. The number of $1 \times 10^{5}$ MD-DCs pulsed with PPD (PPD-DC), BCG (BCG-DC) or LPS (LPS-DC) were incubated for $24 \mathrm{~h}$ and 5 days with autologous $1 \times 10^{6}$ naive CD45RA $\mathrm{R}^{+} \mathrm{T}$ cells or unseparated lymphocytes. The cocultures were performed in 48 well flat-bottomed culture plates ( $1 \mathrm{ml} /$ well). The levels of IL-5 and IFN- $\gamma$ (Diaclone) in supernatants were measured by means of ELISA (sensitivity of both assays was $5 \mu \mathrm{g} / \mathrm{ml}$ ).

Statistical analysis. Data were analyzed using the Statistica 5.0 PL (Statsoft, Poland) A comparison between the prevalence of immune response of naive T cells and total lymphocyte population was made using the Chi-square test. Differences between the responses of naive helper $\mathrm{T}$ cells and unseparated lymphocytes were tested non-parametrically by the Mann-Whitney test. P values of 0.05 or less were considered statistically significant.

\section{Results and discussion}

In the present study we analyzed the immune responses of peripheral blood naive helper $\mathrm{T}$ cells and total lymphocyte population, stimulated with mycobacterial antigen-pulsed DC. Immature MD-DCs from healthy $B C G$ vaccinated volunteers were unpulsed or pulsed with PPD (PPD-DC), live BCG bacilli (BCG-DC) or with LPS (LPS-DC), for the positive control. Then, DCs were cocultured with autologous naive $T$ cells or unseparated lymphocytes, at the ratio of 1:10. The concentration of IFN- $\gamma$ and IL-5 was measured in supernatants collected after $24 \mathrm{~h}$ or at day 5 , by an ELISA assays. Unpulsed MD-DCs incubated with autologous naive $T$ cells or total lymphocytes did not induce any secretion of IFN- $\gamma$.

In the first series of experiments we investigated the prevalence and the production of IFN- $\gamma$ by naive $\mathrm{T}$ 
Table 1. Prevalence of IFN- $\gamma$ production by peripheral blood naive helper T cells and total lymphocyte population to dendritic cells pulsed with PPD, BCG or LPS (24h and 96h cultures).

\begin{tabular}{|l|c|c|}
\hline Antigen & Naive T cells & Total lymphocyte population \\
\hline \multicolumn{3}{|c|}{24 hour-culture } \\
\hline PPD & $21 / 23(91 \%)$ & $15 / 15(100 \%)$ \\
\hline BCG & $13 / 23(56.5 \%)$ & $11 / 15(73.3 \%)$ \\
\hline LPS & $23 / 23(100 \%)$ & $15 / 15(100 \%)$ \\
\hline \multicolumn{3}{|c|}{96 hour-culture } \\
\hline PPD & $23 / 23(100 \%)$ & $15 / 15(100 \%)$ \\
\hline BCG & $23 / 23(100 \%)$ & $15 / 15(100 \%)$ \\
\hline LPS & $23 / 23(100 \%)$ & $15 / 15(100 \%)$ \\
\hline
\end{tabular}

cells and total lymphocytes. IFN- $\gamma$ is a central mediator of cellular Th1 immunity and required for effective immunity against intracellular pathogens including mycobacteria. In addition, IFN- $\gamma$ plays an important role in the activation of APC leading to the development of antibacterial cellular responses [16]. In recent years DC-derived IFN- $\gamma$ has been described as a cytokine participating in a complex autocrine activation loop after mycobacterial stimulation [17]. In the previous work, we showed that IFN- $\gamma$ was successfully produced by naive $\mathrm{T}$ cells stimulated with BCGpulsed DC even when BCG was in the milieu of allergen [18]. Our current study has revealed that very early, in $24 \mathrm{~h}$ cultures, naive helper $\mathrm{T}$ cells from almost all $(91 \%)$ healthy BCG-vaccinated donors produced IFN- $\gamma$ in response to DC pulsed with PPD (Table 1). There was no difference in the prevalence of PPD-DC - driven production of IFN- $\gamma$ by naive helper T cells and total lymphocyte population. Thus, naive helper $\mathrm{T}$ cells in BCG-vaccinated volunteers are in a position to respond by IFN- $\gamma$ to mycobacterial proteins. It is worth mentioning that in a mouse model of $M$. bovis BCG vaccination, $\mathrm{CD}^{+}$cells expressing a resting/naive phenotype were capable of protecting the recipients from a virulent challenge infection, suggesting the emergence of T-cell memory within this subset [19]. As it could be expected, the intensity of IFN- $\gamma$ production by naive helper $T$ cells was significantly lower than by total lymphocyte population (Fig.1). In BCGvaccinated volunteers, unseparated peripheral blood lymphocytes comprise several cell subsets producing IFN- $\gamma$ in response to PPD: naive and memory T cells, $\mathrm{CD} 4^{+}$and $\mathrm{CD} 8^{+}$lymphocytes. The involvement of $\gamma / \delta$ $\mathrm{T}$ cells, natural killer (NK) cells as well as NKT cells can not be excluded. Additionally, recent reports suggest that IFN- $\gamma$ might also be produced by human monocyte-derived DC stimulated by Mycobacterium bovis BCG [17]. The level of IFN- $\gamma$ in the PPD-DC stimulated cultures of naive helper $\mathrm{T}$ cells as well as
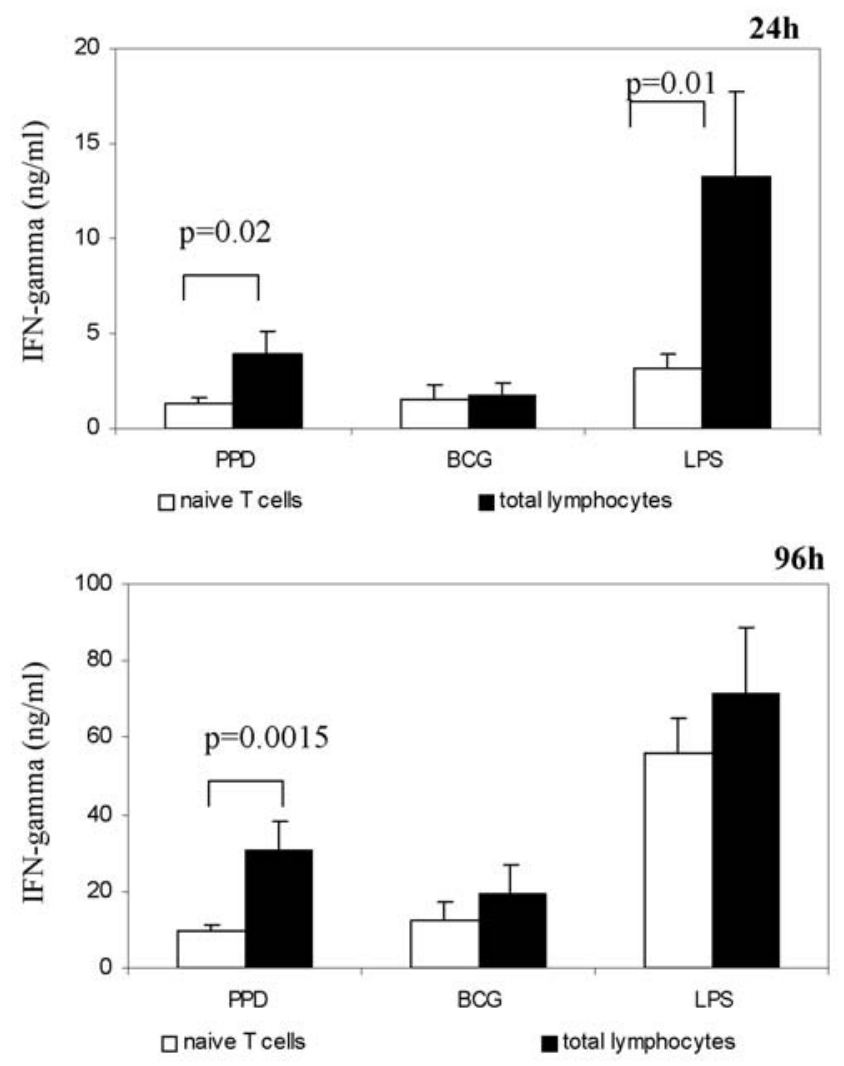

Fig. 1. The intensity of IFN- $\gamma$ production by naive T cells (unfilled columns) and total lymphocyte population (filled columns) in response to PPD, BCG and LPS-pulsed MD-DCs. Immature MDDCs at day 6 of culture were washed, adjusted $1 \times 10^{5}$ cell $/ \mathrm{ml}$, stimulated with PPD, BCG at ratio 1:1 (DC:BCG) or LPS for $24 \mathrm{~h}$ and cultured with $1 \times 10^{6}$ cell $/ \mathrm{ml}$ autologous naive $\mathrm{T}$ cells or total lymphocyte population. Supernatants were examined for IFN- $\gamma$ concentration by ELISA. Values indicate the mean \pm SEM. Detection limit of the assay, $5 \mu \mathrm{g} / \mathrm{ml} ;{ }^{*} \mathrm{p} \leq 0.05$.

total lymphocyte population increased significantly between $24 \mathrm{~h}$ and $96 \mathrm{~h}$ of incubation (Fig.1). Presumably, it was a result of cytokine cross talk between the cells that has been known to be very important during an effector phase of cellular immunity. Thus, NK cells could be activated on antibody dependent pathway. In the previous study we showed [20] that healthy BCGvaccinated subjects produced anti-PPD IgG.

Although, all mentioned leukocyte subsets could theoretically produce IFN- $\gamma$ when stimulated with live $M$. bovis BCG bacilli, we could see no significant difference between the levels of this cytokine in the cultures of unseparated lymphocytes and naive helper $\mathrm{T}$ cells cocultured with BCG-pulsed DC (Fig. 1). It has suggested, that IFN- $\gamma$ measured in unseparated lymphocyte cultures stimulated with BCG-DC was produced mainly by naive $\mathrm{CD} 4^{+}$lymphocytes. Our results are not sufficient to speculate if a similar reactivity to live $M$. bovis BCG bacilli could take place in vivo, in healthy BCG-vaccinated subjects. On the other hand, a lack of the difference in the intensity of IFN- $\gamma$ produc- 
Table 2. Prevalence of IL-5 production by peripheral blood naive helper $\mathrm{T}$ cells and total lymphocyte population to dendritic cells pulsed with PPD, BCG or LPS (24h and 96h cultures).

\begin{tabular}{|l|c|c|}
\hline Antigen & Naive T cells & Total lymphocyte population \\
\hline \multicolumn{3}{|c|}{24 hour-culture } \\
\hline PPD & $2 / 15(13.3 \%)$ & $3 / 10(30 \%)$ \\
\hline BCG & $1 / 15(6.7 \%)$ & $1 / 10(10 \%)$ \\
\hline LPS & $0 / 15(0 \%)$ & $0 / 10(0 \%)$ \\
\hline \multicolumn{3}{|c|}{96 hour-culture } \\
\hline PPD & $15 / 15(100 \%)$ & $10 / 10(100 \%)$ \\
\hline BCG & $15 / 15(100 \%)$ & $7 / 10(70 \%)$ \\
\hline LPS & $14 / 15(93.3 \%)$ & $6 / 10(60 \%)$ \\
\hline
\end{tabular}

tion in BCG-DC - stimulated cultures of naive helper $\mathrm{T}$ cells and total lymphocyte population could reflect an activation of $\mathrm{T}$ regulatory cells expressing memory phenotype by whole $M$. bovis BCG bacilli, in unseparated lymphocytes. As it has been showed by Li et al. [21] the increased frequency of $\mathrm{CD} 4{ }^{+} \mathrm{CD} 25^{\text {high }}$ cells inhibit BCG-specific induction of IFN- $\gamma$ by $\mathrm{CD} 4^{+} \mathrm{T}$ cells from TB patients.

The $E$. coli LPS was included into the study as a control. An intensive production of IFN- $\gamma$ was observed early, at $24 \mathrm{~h}$, in the cultures of unseparated lymphocytes stimulated with LPS-pulsed DC. The cytokine production presumably resulted from the stimulation of various cell subsets through numerous cell-signaling proteins and transcriptional factors. Interestingly, naive helper $\mathrm{T}$ lymphocytes produced also a certain amount of IFN- $\gamma$ when stimulated with LPS-DC for 24 hours, although significantly less than unseparated lymphocytes (Fig. 1). In 96h cultures with LPS-pulsed DC, an equally intensive production of IFN- $\gamma$ was observed for the cultures of unseparated lymphocytes and naive helper T cells. An involvement of DCs in the synthesis of IFN- $\gamma$ in LPS-DC cultures seems quite probable.

Our study has demonstrated that M. bovis BCG bacilli and PPD as a mycobacterial protein activate naive cells of a Th1 lineage from healthy BCG-vaccinated subjects. It is generally accepted that the immunity to tuberculosis relies mainly on the functions of Th1 rather than Th2 helper lymphocytes. However, the BCG vaccine is at best credited with a $50 \%$ overall protective efficacy [22]. Recently, it has been suggested that IFN- $\gamma$ response may not be enough for the protection against tuberculosis and an IFN- $\gamma$ - negative cell population capable of rapid activation in an emergency also persists in BCG-vaccinated subjects [19]. Taking that statement into consideration, we decided to estimate the production of IL-5 as a Th2-type cytokine by



Fig 2. The intensity of IL-5 production by naive T cells (unfilled columns) and total lymphocyte population (filled columns) in response to PPD, BCG and LPS-pulsed MD-DCs. Immature MDDCs at day 6 of culture were washed, adjusted $1 \times 10^{5}$ cell $/ \mathrm{ml}$, stimulated with PPD, BCG at ratio 1:1 (DC:BCG) or LPS for $24 \mathrm{~h}$ and cultured with $1 \times 10^{6}$ cell $/ \mathrm{ml}$ autologous naive $\mathrm{T}$ cells or total lymphocyte population. Supernatants were examined for IL-5 concentration by ELISA. Values indicate the mean SEM . Detection limit of the assay, $5 \mu \mathrm{g} / \mathrm{ml} ;{ }^{*} \mathrm{p} \leq 0.05$.

naive helper cells and total lymphocyte population from BCG-vaccinated donors, stimulated with DC pulsed with mycobacterial antigens. The lymphocytes cocultured with unpulsed autologous DCs did not secrete IL-5 indicating that the production of this cytokine was antigen-dependent. In $24 \mathrm{~h}$ cultures the prevalence and intensity of IL-5 production were very low (Table 2). On the contrary, in 96h-cultures, naive helper $\mathrm{T}$ cells from all donors produced IL-5 when stimulated with PPD-DC or BCG-DC (Fig. 2). Moreover, there was no significant difference between the IL-5 levels in the cultures of naive helper T cells and unseparated lymphocytes stimulated with PPD-DC or BCG-DC. PPD-DC driven IL-5 production was significantly more intensive than BCG-induced release of IL-5, in naive helper T cells $(\mathrm{p}=0.02)$ and in total lymphocyte population $(\mathrm{p}=0.04)$.

Our present results clearly showed that circulating naive $\mathrm{CD} 45 \mathrm{RA}^{+} \mathrm{CD}^{+}$lymphocytes from BCG-vaccinated volunteers can become effector helper cells producing both IFN- $\gamma$ and IL-5 under the stimulation by autologous dendritic cells presenting mycobacterial protein antigen-PPD or infected with live $M$. bovis BCG bacilli. The kinetics and intensity of generation of Th1 and Th2 responses in dendritic cell model depend on the type of presented antigen. The PPD-DC and BCG-DC driven IFN- $\gamma$ generation preceded the release of IL-5. A delay in IL-5 production could result from an inhibition of the proliferation of Th2 cells by IFN- $\gamma$. But we cannot rule out the possibility that Th1/Th2 balance is critical for the control of cellular responses to Mycobacteria.

Acknowledgments: This work was supported by grant of Ministry of Science and Higher Education in Poland No 2PO5A 15628 


\section{References}

[ 1] Clark GJ, Angel N, Kato M, et al. The role of dendritic cells in the innate immune system. Microbes and Infect. 2000;2:257-272.

[ 2] Banchereau J, Briere F, Caux C et al. Immunobiology of dendritic cells. Annu Rev Immunol. 2000;18:767-811.

[3] Langenkamp A, Messi M, Lanzavecchia A, Sallusto F. Kinetics of dendritic cell activation: impact on priming of Th1, Th2 and nonpolarized T cells. Nat Immunol. 2000;1:311-316.

[4] Palucka K, Banchereau J. How dendritic cells and microbes interact to elicit or subvert protective immune responses. Curr Opin Immunol. 2002;14:420-31.

[5] O'Garra A. Cytokines induce the development of functionally heterogeneous T helper cell subsets. Immunity. 1998;8:275283.

[ 6] Weaver CT, Harrington LE, Mangan PR, Gavrieli M, Murphy KM. Th17: An effector CD4 T cell lineage with regulatory T cell ties. Immunity. 2006;24:677-688.

[ 7] Kamijo R, Le J, Shapiro D et al. Mice that lack the interferon-gamma receptor have profoundly altered responses to infection with Bacillus Calmette-Guerin and subsequent challenge with lipopolysaccharide. J Exp Med. 1993;178:14351440.

[ 8] Girard MP, Fruth U, Kieny MP. A review of vaccine research and development: Tuberculosis. Vaccine. 2005;23:5725-5731

[ 9] Demangel C, Bean AG, Martin E, Feng CG, Kamath AT, Britton WJ. Protection against aerosol Mycobacterium tuberculosis infection using Mycobacterium bovis Calmette-Guerininfected dendritic cells. Eur J Immunol. 1999;29:1972-1979.

[10] Jiao X, Lo-Man R, Guermonprez et al. Dendritic cells are host cells for mycobacteria in vivo that trigger innate and acquired immunity. J Immunol. 2002;168:1294-1301.

[11] Hope JC. Thom ML, McCormick PA, Howard CJ. Interaction of antigen presenting cells with mycobacteria. Vet Immunol Immunopathol. 2004;100:187-195.

[12] Demangel C, Britton WJ. Interaction of dendritic cells with mycobacteria: where the action starts. Immunol Cell Biol. 2000;78:318-324.
[13] Tsuji S, Matsumoto M, Takeuchi O et al. Maturation of human dendritic cells by cell wall skeleton of Mycobacterium bovis bacillus Calmette-Guérin: involvement of Toll-like receptors. Infect Immun. 2000;68:6883-6890.

[14] Tala-Heikkila MM, Tuominen JE, Tala EO. Bacillus Calmette-Guérin revaccination questionable with low tuberculosis incidence. Am J Respir Crit Care Med. 1998;157:13241327.

[15] Kelly P, McKeown D, Clancy L. Neonatal BCG vaccination in Ireland evidence of its efficacy in the prevention of childhood tuberculosis. Eur Respir J. 1997;10:619-623.

[16] Shtrichman R, Samuel CE. The role of $\gamma$ interferon in antimicrobial immunity. Curr Opin Microbiol. 2001;4:251-259.

[17] Fricke I, Mitchell D, Mittelstadt J, Lehan N et al. Mycobacteria induce IFN-gamma production in human dendritic cells via triggering of TLR2. J Immunol. 2006;176:5173-5182.

[18] Kowalewicz-Kulbat M, Pestel J, Biet F, Locht C et al. Myobacterium bovis BCG Mycobacteria-New Application. Pol J Microbiol. 2006;55:13-17.

[19] Kipnis A, Irwin S, Izzo AA, Basaraba IR, Orme IM. Memory $\mathrm{T}$ lymphocytes generated by Mycobacterium bovis BCG vaccination reside within a CD4 CD44lo CD62 ligandhi population. Infect Immun. 2005;73:7759-7764.

[20] Paziak-Domańska B, Bonar A, Kowalewicz-Kulbat M. et al. The lack of relationship between serum content of MBL, sCD14, antiPPD and anti-Hsp65 IgG and ingestion of Mycobacterium bovis BCG bacilli by phagocytes. Arch Immun Ther Exp. 2002;50:337-344.

[21] Li L, Lao S-h, Wu Ch-y. Increased frequency of CD4+CD25 high cells inhibit BCG-specific induction of IFNby CD4+ T cells from TB patients. Tuberculosis. 2007; (article in press).

[22] Brewer TF, Colditz GA. Relationship between Bacille Calmette-Guérin (BCG) strains and the efficacy of BCG vaccine in the prevention of tuberculosis. Clin Infect Dis. 1995;20: 126-135.

Submitted: 28 September 2007 Accepted after reviews: 15 February, 2008 